\title{
Risk factors of stunting and wasting in Somali pre-school age children: results from the 2019 Somalia micronutrient survey
}

William E. S. Donkor ${ }^{1}$, Joshua Mbai², Fatmata Sesay ${ }^{3}$, Sundus Ibrahim Ali², Bradley A. Woodruff ${ }^{1}$, Shuaib Mohamoud Hussein ${ }^{2}$, Kheyriya Mohamed Mohamud ${ }^{4}$, Ahmed Muse ${ }^{5}$, Warsame Said Mohamed ${ }^{6}$, Abdullahi Muse Mohamoud ${ }^{4}$, Farhan Mohamed Mohamud ${ }^{4}$, Nicolai Petry' ${ }^{1}$, Melanie Galvin ${ }^{3}$, Rita Wegmüller ${ }^{1}$, Fabian Rohner ${ }^{1}$, Yvonne Katambo ${ }^{2}$ and James P. Wirth ${ }^{1^{*}}$

\begin{abstract}
Background: Stunting and wasting in children less than 5 years of age are two key indicators of child malnutrition. Reducing their prevalence is a priority of the global public health community and for Somalia, a country suffering complex humanitarian emergencies such as drought, flooding, conflict and large-scale displacements.

Methods: Data from the nationally representative cross-sectional Somalia Micronutrient Survey (SMS 2019) on 1947 children were analyzed to assess the prevalence and potential risk factors of stunting and wasting. Bivariate and multivariable analyses were conducted separately for children 0-5 months and 6-59 months, and population attributable fractions were calculated using adjusted risk ratios produced by Poisson regression models.

Results: Among the 1947 children, the prevalence of stunting and wasting were $17.2 \%$ (95\% Cl: 15.0, 19.6) and 11.0\% (95\% Cl: 9.3, 12.9), respectively. Among children 6-59 months of age, those residing in severely food insecure households had a higher risk of stunting (adjusted risk ratio [aRR] 1.47; Cl: 1.12, 1.93) compared to those in food secure households. This risk of stunting was also higher in children with inflammation (aRR 1.75; Cl: 1.35, 2.25) and iron deficiency (ID) (aRR 2.09; Cl: 1.58, 2.80). For wasting, a dose-response relationship was found with household wealth, with the risk of wasting increasing significantly as the household wealth quintile decreased. On the other hand, the risk of wasting was lower in iron-deficient children (aRR 0.69; Cl: 0.49, 0.98) than in iron-replete children. Among children $0-5$ months of age no variables remained statistically significantly associated with stunting in the multivariable analysis. Wasting, however, was more common in children with recent diarrhea (aRR 3.51; Cl: 1.68, 7.36).
\end{abstract}

Conclusions: Nutritional status of children in Somalia may be improved by prevention of diarrhea and other infections and improvements in household food security.

Keywords: Stunting, Wasting, Micronutrients, iron, Children, Somalia, Risk factors, Malnutrition

\section{Background}

Stunting and wasting are two key nutrition indicators in children $<5$ years of age. Globally, stunting affected $22.0 \%$ and wasting $6.7 \%$ of children under 5 years of age in 2020 , and Africa has one of the highest stunting and wasting prevalence, only second to Asia [1]. 
Stunting is a result of suboptimal nutrition or longterm nutrition deprivation, which can occur in utero and during childhood $[1,2]$. Short-term consequences of stunting include increased risk of infectious diseases [2], poor cognitive development [3], and increased morbidity [4]. The long-term consequences of stunting include reduced height and lean body mass in adulthood, along with decreased cognitive performance between 6 and 11 years of age and less educational attainment overall [5].

Wasting is due to inadequate nutrient intake and/or disease [6]. Wasted children have an acutely increased risk of death [1,7] and therefore require urgent medical and nutrition treatment [7]. Wasting in young children often results in a weakened immunity and delayed physical development [1].

The prevalence of stunting and wasting in Africa currently stands at 41 and $27 \%$ respectfully. Regionally, the prevalence of stunting and wasting in Eastern Africa, Somalia included, has declined steadily from the year 2000 with the percentage of children stunted and wasted currently at 32.6 and $5.2 \%$ respectively. Stunting and wasting may co-exist [1], and the burden is highest in areas with ongoing conflicts [8]. In Somalia, humanitarian emergencies due to natural disasters and civil conflict put children below 5 years of age at particular risk of malnutrition. While monitoring of nutrition indicators is difficult under such circumstances [9], biannual cross-sectional surveys that include anthropometric measurements of children are often conducted in selected areas of Somalia [10]. Using pooled cross-sectional surveys from 2007 and 2010, researchers found that about 31 and $21 \%$ of children $6-59$ months of age were stunted and wasted, respectively [6]. Based on this study, stunting and wasting prevalence in Somalia would be classified as "high" and "very high" according to WHO classifications [11]. More recent data from biannual crosssectional surveys were available, but did not present national-level results.

The 2019 Somalia Micronutrient Survey (SMS) [12] was done to provide updated national estimates of stunting, wasting, and other nutritional deficiencies. Using anthropometric data collected from children and data on other household members and household characteristics, we aimed to identify the potential risk factors of stunting and wasting in children $0-5$ months and 6-59 months of age. A detailed understanding of the risk factors of stunting and wasting in both age groups will serve as the basis for policy development and targeted interventions to address childhood undernutrition.

\section{Methods}

\section{Study design}

The 2019 SMS, a nationally-representative stratified cross-sectional household-based survey, was conducted between December 2018 and September 2019. Sampling was done from 6 strata: 1) Somaliland, 2) Puntland, 3) the Somalia states of Hirshabelle and Galmudug; 4) the Somalia states of Jubaland and South-West, 5) the Banaadir administrative region of Somalia, and 6) internally displaced persons (IDPs) settlements in all five aforementioned geographic strata combined.

The survey used a two-stage cluster sampling procedure. Enumeration areas (EAs) and IDP camps were primary sampling units which were selected using probability proportional to size. Within each selected primary sampling unit, the required number of households were selected using simple random sampling. In the first 5 geographic strata, 25 EAs were selected in each stratum after excluding EAs that were not accessible due to insecurity; EAs were categorized as either urban or rural. In the 6th stratum, 25 IDP camps were selected. In each EA and IDP camp, 16 households were randomly selected resulting in a total sample of 2400 households. Further details of the selection procedure can be found elsewhere [12].

Within participating households, all children $0-59$ months of age were eligible to be recruited into the survey, questionnaires in the child questionnaire were answered by the selected child's mother or caretaker. Non-pregnant women were recruited from a randomly selected one-half subsample.

\section{Questionnaire data}

Data on household characteristics were collected from the household head or knowledgeable adult household member. The household interview collected information on the household's dwelling, durable goods ownership, water source, and sanitation facility using standard questions widely used in health and nutrition assessment surveys worldwide. Durable goods and dwelling characteristics were used to calculate a household wealth index using the standard methods [13, 14]. Household-level sanitation facilities and water source were respectively classified as adequate/inadequate or safe/unsafe based on WHO/UNICEF guidelines [15]. To estimate sanitation status at the community-level, the proportion of households in each cluster with inadequate sanitation facilities was calculated and categorized into sub-groups of $0-19 \%, 20-39 \%, 40-59 \%, 60-79 \%$, and $80-100 \%$. In addition, household food security was assessed using the Household Food Insecurity Access Scale (HFIAS) questionnaire module. The final scale was categorized into 
four categories: food secure, mild food insecurity, moderate food insecurity, and severe food insecurity [16].

The child questionnaire collected data on age, sex, recent morbidity, and consumption of vitamin and mineral supplements. Child age was calculated by subtracting the child's date of birth from the date of the interview. As much of the population in Somalia was not calendar literate, a local event calendar for the past 5 years was developed to facilitate the approximation of the child's birth date. The child questionnaire included standard infant and young child feeding questions [17] that were administered to children 0-23 months of age. Questionnaire data was also collected from women, however, as women were only recruited from $1 / 2$ of all households, maternal-level data was not included in this analysis. The Open Data Kit (ODK) software was used for direct electronic questionnaire data entry.

\section{Anthropometric assessments}

Anthropometrists were trained in standard anthropometric techniques [18]. Performance during a standardization exercise, the results of a post-test, and observations from trainers were used to select the best performing team members. All survey procedures were practiced in a field test under close supervision prior to the start of data collection.

During the survey field work, the weight and height or length of each child was measured according to standard procedures [19]. Child weight was measured using an automatically taring bathroom scale $\left(\mathrm{SECA}^{\circledR}\right.$, Hamburg, Germany); all scales were calibrated each morning before the start of data collection. The standard wooden height board (UNICEF item number S0114540, Copenhagen, Denmark) was used to measure the child height or length. Length/height and weight measurements were taken twice by the same measurer and both values recorded. The second length/height measurement was taken after the child was removed and replaced on the height board to account for incorrect positioning during the first height measurement. For weight measurements, children that could not stand were directly weighed on the scale, and the scale's tare function was used for children that could not stand. For the second measurement, children were either re-weighed directly or the tare process was repeated.

\section{Blood biomarkers}

Capillary blood was collected from all children 6-59 months; no blood was taken from children younger than 6 months of age to prevent potential injury. Blood was collected from the heel in children 6-11 months of age and from the finger in children 12-59 months of age. After sterilization with an alcohol pad, the puncture site was wiped dry and punctured with a "contact activated" high-flow blade lancet (Becton Dickinson, Franklin Lakes, NJ, USA). After wiping the first drop of blood away, the second and third drop of blood were used to measure hemoglobin concentration and recent or current malaria infection, followed by the collection of $300-400 \mu \mathrm{l}$ of capillary blood into a silica-coated blood collection tube (Sarstedt, Microvette ${ }^{\circledR} 300$ Z, Nümbrecht, Germany).

Following on-site measurements of haemoglobin and malaria parasitemia, the labeled microtainers were placed in a cool box at $2-8^{\circ} \mathrm{C}$ in the dark for transport in the evening of the same day to one of four state laboratories. Samples were centrifuged at $3000 \mathrm{rpm}$ for $7 \mathrm{~min}$ to separate the serum, which was then aliquoted into labeled cryovials. Aliquots were stored at $-20^{\circ} \mathrm{C}$ and later shipped on dry ice for analyses. Serum samples were analyzed for retinol-binding protein (RBP), ferritin, C-reactive protein (CRP), and alpha 1-acid glycoprotein (AGP) at the VitMin-Lab (Wilstaett, Germany) using an ELISA method.

\section{Data management and statistical analysis}

Anthropometry, anemia and malaria data were collected on paper forms, and subsequently entered into ODK on the same day. Data analysis was done using Stata/ IC version 14.2. All analyses of questionnaire data were conducted using sampling weights to account for the unequal probability of selection in the six strata.

Z-scores were calculated based on WHO's 2006 Growths Standards [20], and children with height-forage $\mathrm{z}$-scores (HAZ) and weight-for-height $\mathrm{z}$-scores $(\mathrm{WHZ})<-2.0$ standard deviations were classified as stunted and wasted, respectively [20]. Prior to data analysis, the quality of anthropometric measurements was examined for all teams. As a result of this examination, child height measurements taken by all teams working in Galmudug State were excluded, and length, height, and weight measurements taken by one team in South-West State and one team in Jubaland State were excluded [12]. Further details about anthropometric data quality are presented elsewhere [12].

Any inflammation was defined as concentrations of CRP $>5 \mathrm{mg} / \mathrm{L}$ and/or concentrations of AGP $>1 \mathrm{~g} / \mathrm{L}$ [21]. After adjusting serum ferritin and RBP concentrations for inflammation using the Biomarkers Reflecting Inflammation and Nutritional Determinants of Anemia (BRINDA) approach, iron deficiency (ID) was defined as serum ferritin concentrations $<12 \mu \mathrm{g} / \mathrm{L}$ [22] and vitamin A deficiency was defined as RBP concentrations $<0.7 \mu \mathrm{mol} / \mathrm{L}$ [23].

For categorical variables, proportions were calculated to derive the prevalence. All measures of precision, 
including 95\% confidence limits and chi square $p$ values for differences in subgroup prevalence, were calculated accounting for the complex cluster and stratified sampling used by the SMS 2019.

For this analysis, we identified potential risk factors from the recent WHO conceptual framework for stunting [24] and other potential risk factors of wasting identified from a review of the literature. Bivariate and multivariable analyses were conducted separately for children $0-5$ months and 6-59 months to identify potential risk factors of stunting and wasting. Separate analyses for these age groups were done to account for the fact that blood biomarkers were only available in children $6-59$ months of age. For all analyses, significance was accepted at $P<0.05$.

Variables with chi-square $p$-values $<0.1$ during categorical bivariate analyses were included in the four subsequent multivariable models. When applicable during the bivariate analysis, a nonparametric test for trend was conducted using Stata's nptrend command [25] to identify dose-response relationships. Following tests of collinearity (i.e., variance inflation factor), multivariable Poisson regression models were run using backwards elimination until only variables with statistically significant associations were remaining. To account for age differences, age in months was included in all regression models.

Following the multivariable analyses, the population attributable fraction (PAF) was calculated for all statistically significant potential risk factors. PAF was calculated using the equation $p d\left(\frac{a R R-1}{a R R}\right)$ using the adjusted risk ratios (aRRs) produced by the Poisson regressions and the proportion of cases with the potential risk factor of interest (pd) [26].

\section{Results}

\section{Description of survey sample}

Table 1 shows the demographic characteristics of children included in the survey sample. Of the 1947 children recruited into the survey, approximately $20 \%$ are found in each 1-year age group between 12 and 59 months of age, however, children between 0 and 11 months accounted for only $15.8 \%$ of all children surveyed. The number of males and females were evenly distributed, and approximately half of the children resided in urban areas, onethird in rural areas, and 15\% in IDP settlements.

\section{Distribution of stunting prevalence in children 0-59 months}

In children 0-59 months of age, the stunting prevalence was $17.2 \%$ (95\% CI: 15.0, 19.6), and the weighted mean and standard deviation of HAZ was -0.50 and 1.73 , respectively. The prevalence of stunting is considered
Table 1 Description of sampled children (0-59 months), Somalia 2019

\begin{tabular}{llll}
\hline Characteristic & \multicolumn{3}{l}{ Survey Sample } \\
\cline { 2 - 4 } & $\mathbf{n}$ & $\mathbf{\%}^{\mathbf{a}}$ & $\mathbf{( 9 5 \% ~ C l )}$ \\
\hline Age Group (in months) & & & \\
$0-5$ & 136 & 7.3 & $(5.8,9.1)$ \\
$6-11$ & 165 & 8.5 & $(7.3,9.8)$ \\
$12-23$ & 386 & 19.7 & $(17.7,21.8)$ \\
$24-35$ & 434 & 22.6 & $(20.8,24.5)$ \\
$36-47$ & 436 & 22.3 & $(20.3,24.3)$ \\
$48-59$ & 390 & 19.8 & $(17.9,21.8)$ \\
Sex & & & \\
Male & 979 & 50.9 & $(48.5,53.4)$ \\
Female & 968 & 49.1 & $(46.6,51.5)$ \\
Residence & & & \\
Rural & 626 & 31.9 & $(23.9,41.0)$ \\
Urban & 1042 & 53.2 & $(44.4,61.8)$ \\
IDP & 279 & 15.0 & $(12.7,17.6)$ \\
ALL CHILDREN & 1947 & $100.0 \%$ & - \\
\hline
\end{tabular}

Note: The n's are un-weighted numbers of subjects in each subgroup; the sum of subgroups may not equal the total because of missing data

a Percentages are un-weighted and do not account for unequal probability of selection

${ }^{\mathrm{b}} \mathrm{Cl}$ Confidence interval, calculated taking into account the complex sampling design

a medium public health problem according to WHO's criteria [27]. As shown in panel A of Fig. 1, the HAZ distribution is shifted to the left of the WHO Growth Standards, illustrating that children in Somalia are slightly shorter than children from healthy reference populations [20]. Moderate and severe stunting were found in $10.5 \%$ (95\% CI: $8.9,12.3)$ and $6.7 \%$ (95\% CI: $5.4,8.3)$ of children in this age group, respectively. The prevalence of stunting was higher among male children (19.3\%; 95\% CI: 16.1, 23.0) than female children (15.0\%; 95\% CI: 12.3 , $18.1)$, although this difference was not strictly statistically significant $(p=0.055)$.

Distribution of wasting prevalence in children 0-59 months In children 0-59 months of age, the prevalence of wasting was $11.0 \%$ (95\% CI: 9.3, 12.9), denoting a serious public health problem according to the WHO thresholds [27]. The mean and standard deviation of WHZ were -0.48 and 1.35, respectively. As shown in panel B of Fig. 1, the WHZ is shifted to the left of the WHO Child Growth Standards [20]. Severe wasting was found in fewer children $(2.3 \%$; 95\% CI: $1.7,3.2)$ than moderate wasting (8.6\%; 95\% CI: 7.1, 10.4). The prevalence of wasting was higher among male children (12.2\%; 95\% CI: 9.8, 15.1) than female children $(9.7 \%$; $95 \%$ CI: $7.7,12.1)$, but the difference was not statistically significant $(p=0.12)$. 


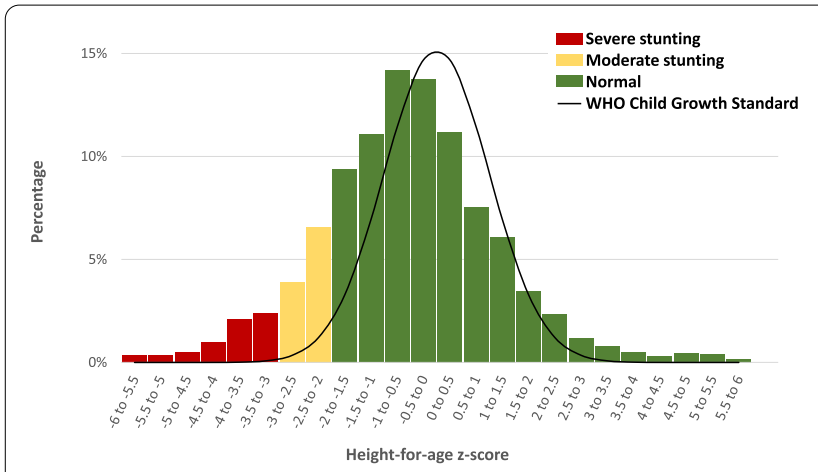

A

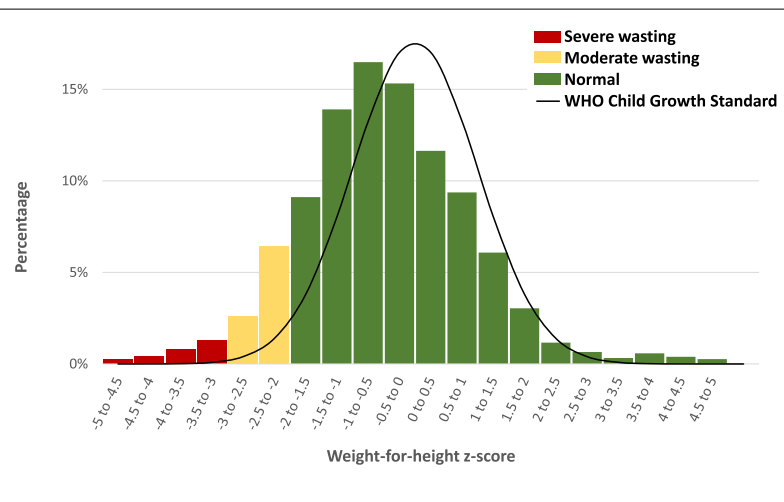

B

Fig. 1 Distribution of height-for-age z-scores (A) and weight-for-height z-scores (B), children 0-59 months of age, Somalia 2019

\section{Bivariate associations between stunting and various potential risk factors in children $\mathbf{0 - 5}$ months and 6-59 months of age}

The prevalence of stunting in children $0-5$ and $6-59$ months of age by various potential risk factors is provided in Supplementary Table S1.

The prevalence of stunting in children $0-5$ months of age was $10.9 \%$ (95\% CI: 6.1, 18.7). Only one potential risk factor showed a statistically significant association with stunting in the bivariate analysis. The prevalence of stunting was unexpectedly higher in children residing in households with adequate sanitation facilities compared to children in households with inadequate sanitation facilities ( $17.1 \%$ vs $2.8 \%$, respectively; $p<0.005)$.

The prevalence of stunting in children 6-59 months of age was $17.8 \%$ (95\% CI: $15.5,20.3)$. Stunting was significantly associated with age $(p<0.001)$, with the highest prevalence found among children $24-35$ months of age (23\%) and the lowest prevalence (11.5\%) found in both the youngest and the oldest age groups. Stunting prevalence was higher in IDP settlements compared to rural and urban settlements $(28.4,13.8$, and $16.5 \%$, respectively; $p<0.01)$. In addition, stunting prevalence was substantially higher in South-West State $(38.9 \% ; p<0.001)$ than in other states; the next highest prevalence of stunting was $25.6 \%$ in Banaadir. The prevalence of stunting showed a progressive decline with increasing household wealth $(p<0.005)$, from $33.9 \%$ in the poorest quintile to $11.5 \%$ in the richest quintile. The nonparametric test for trend found a statistically significant $(p<0.001)$ decrease in stunting prevalence by increasing household wealth. The stunting prevalence among children with inflammation was almost double the prevalence in children with no inflammation $(26.1 \%$ vs $13.8 \% ; p<0.001)$. Children in households in which soap was available had a lower prevalence of stunting than children in households in which soap was not available $(14.6 \%$ vs. $22.7 \% ; p<0.01)$. The stunting prevalence showed a progressive increase with increasing food insecurity, from $13.5 \%$ in children in food secure households to $21.8 \%$ in children in households with severe food insecurity $(p<0.01)$. A statistically significant increase $(p<0.001)$ in the prevalence of stunting as by food insecurity group was observed with the nonparametric test for trend.

\section{Multivariable analysis - risk factors of stunting in children 0-5 months and 6-59 months of age}

As a first step in the model building process, collinearity between the potential risk factors was explored by calculating the variance inflation factor for the preliminary models. Variance inflation factor results were consistently $<2$ indicating that there was no collinearity.

Table 2 presents the final parsimonious multivariable models for stunting in children $0-5$ and 6-59 months of age. In children $0-5$ months, no variable remained statistically significant for stunting in the model. In contrast, in older children, several variables remain statistically significantly associated with stunting. Iron deficiency accounted for approximately one-third of stunting in this age group, while inflammation and severe household food insecurity accounted for less than $20 \%$. Unlike severe household food insecurity, mild and moderate food insecurity were not statistically significantly associated with stunting.

\section{Bivariate associations between wasting and various potential risk factors in children 0-5 months and 6-59 months of age}

The wasting prevalence for various potential risk factors in children $0-5$ and $6-59$ months of age by is provided in Supplementary Table S2. 
Table 2 Multivariable analysis: Adjusted relative risk and population attributable fraction of stunting and wasting in children 0-5 months and 6-59 months, Somalia 2019

\begin{tabular}{|c|c|c|c|c|}
\hline Characteristic & Category & Adjusted relative risk & $95 \% \mathrm{Cl}$ & $\begin{array}{l}\text { Population } \\
\text { attributable } \\
\text { fraction }^{b}\end{array}$ \\
\hline \multicolumn{5}{|l|}{$0-5$ months $^{a}$} \\
\hline \multicolumn{5}{|l|}{ No variables significant } \\
\hline \multicolumn{5}{|l|}{$6-59$ months $(n=1179)^{a}$} \\
\hline \multirow[t]{2}{*}{ Inflammation ${ }^{c}$} & Yes & 1.75 & $(1.35,2.25)$ & $16.9 \%$ \\
\hline & No & referent & & \\
\hline \multirow[t]{2}{*}{ Iron status $^{d}$} & Deficient & 2.09 & $(1.58,2.80)$ & $34.6 \%$ \\
\hline & Sufficient & referent & & \\
\hline \multirow[t]{4}{*}{ Household food security status } & Severe food insecurity & 1.47 & $(1.12,1.93)$ & $17.1 \%$ \\
\hline & Moderate food insecurity & 1.33 & $(0.88,2.03)$ & $2.3 \%$ \\
\hline & Mild food insecurity & 0.86 & $(0.44,1.69)$ & $-0.7 \%$ \\
\hline & Food secure & referent & & \\
\hline \multicolumn{5}{|l|}{$0-5$ months $(n=102)^{a}$} \\
\hline \multirow[t]{2}{*}{ Diarrhea in past 2 weeks } & Yes & 3.51 & $(1.68,7.36)$ & $28.0 \%$ \\
\hline & No & referent & & \\
\hline \multirow[t]{2}{*}{ Exclusive breastfeeding ${ }^{e}$} & Yes & 0.00 & $(0.00,0.00)$ & - \\
\hline & No & referent & & \\
\hline \multicolumn{5}{|l|}{$6-59$ months $(n=1178)^{a}$} \\
\hline \multirow[t]{2}{*}{ Iron status $^{d}$} & Deficient & 0.69 & $(0.49,0.98)$ & $-18.2 \%$ \\
\hline & Sufficient & referent & & \\
\hline \multirow[t]{5}{*}{ Household wealth quintile } & Lowest & 2.24 & $(1.02,4.87)$ & $4.5 \%$ \\
\hline & Second & 2.21 & $(1.27,3.82)$ & $10.7 \%$ \\
\hline & Middle & 1.95 & $(1.23,3.10)$ & $14.5 \%$ \\
\hline & Fourth & 1.33 & $(0.81,2.21)$ & $5.7 \%$ \\
\hline & Highest & Referent & - & \\
\hline
\end{tabular}

${ }^{a}$ Age in months as a continuous variable included in all models. ${ }^{b}$ Population attributable fraction calculated using adjusted relative risk from Poisson regression. Inflammation defined as elevated CRP ( $>5 \mathrm{mg} / \mathrm{L}$ ) and/or elevated AGP ( $>1 \mathrm{~g}(\mathrm{~L})$. Iron deficiency defined as inflammation-adjusted (using BRINDA approach) serum ferritin $<12 \mu \mathrm{g} / \mathrm{L}$. ${ }^{\mathrm{e}}$ Relative risk of wasting for exclusive breastfeeding was nearly zero, with relative risk and associated confidence intervals represented in scientific notation (2.92e-08; $95 \% \mathrm{Cl}: 1.51 \mathrm{e}-08,5.64 \mathrm{e}-08)$

The prevalence of wasting in children $0-5$ months of age was $17.2 \%$ (95\% CI: 9.2, 30.0). Of the various potential risk factors explored in this age group, only diarrhea and exclusive breastfeeding were significantly associated with wasting. The wasting prevalence was significantly higher among children who had diarrhea in the past 2 weeks compared to those without diarrhea $(53.3 \%$ vs. $12.0 \%$; respectively; $p<0.001$ ). Wasting prevalence was also lower among exclusively-breastfed children compared to those not exclusively breastfed ( $0.0 \%$ vs $21.7 \%$, respectively; $p=0.093$ ).

The prevalence of wasting in children 6-59 months of age was $10.5 \%$ (95\% CI: 8.9, 12.4). Wasting was significantly associated with age $(p<0.05)$, children $48-59$ months of age had the highest wasting prevalence $(15.0 \%)$. State of residence $(p<0.01)$ was also significantly associated with wasting. The highest prevalence, found in Jubaland (15.9\%), constitutes a severe public health problem according to WHO classification standards [27].
Household wealth was significantly associated with wasting, with a suggestion of a decline in the prevalence with increasing household wealth. Although the wasting prevalence did not decrease for all household wealth groups (i.e., wasting increased from $11.7 \%$ in the poorest group to $16.4 \%$ in second group), the nonparametric test for trend showed a statistically significant $(p<0.01)$ decrease in wasting as wealth group increased. Children with LRI in the past 2 weeks had significantly higher levels of wasting compared to children with no LRI $(16.4 \%$ vs. $10.1 \%$; $p<0.05)$.

\section{Multivariable analysis - risk factors of wasting in children 0-5 months and 6-59 months of age}

Prior to backwards elimination, variance inflation factor calculations were conducted for the preliminary models that contained all variables associated with wasting at $p<0.1$ during the bivariate analyses. Variance inflation factor results were consistently $<2$ for all risk factors in 
all models, showing no collinearity in the preliminary models.

In children $0-5$ months of age, two variables were strongly associated with wasting: recent diarrhea and exclusive breastfeeding. Children with a history of recent diarrhea were more than 3 times as likely to be wasted as children without such a history, and approximately $30 \%$ of wasting in this age group was attributable to recent diarrhea. Children $0-5$ months of age who were exclusively breastfed had essentially no risk of being wasted. Due to the lack of variability between wasting and exclusive breastfeeding, no population attributable fraction could be calculated.

In older children, iron deficiency was protective against wasting. As a result, the population attributable fraction is negative. Household wealth showed a dose-response relationship with wasting in this age group; however, the lowest wealth quintiles contributed less to wasting than did the middle wealth quintiles.

\section{Discussion}

\section{Stunting in children 0-5 and 6-59 months of age}

As expected, our study found that factors associated with stunting were different in children 0 to 5 months of age and children 6 to 59 months of age. The lack of significantly associated variables with stunting in children 0 to 5 months of age could be due to the small sample size in our study. This survey's sampling scheme selected children 0 to 5 months of age in proportion to their representation in the larger population of children less than 5 years of age. As a result, they represent about $10 \%$ of our total survey sample of children. Additionally, stunting in this age group may stem predominantly from antenatal factors which were not measured in this survey. In an analysis of data from 137 developing countries, Danaei et al. concluded that fetal growth restriction was the main risk factor of stunting [28]. Few children surveyed in the SMS 2019 had been weighed at birth, and data on estimated birth size - a proxy for being born small for gestational age - was not collected. As our study did not collect blood samples from children 0-5 months of age, we could not examine various biomarkers, such as inflammation, which has been associated with children $<6$ months of age in other countries [29,30] and was associated with stunting in older children in our study.

In children 6-59 months of age, inflammation, household food security status, and ID were the only variables statistically significantly associated with stunting in the final regression model. Inflammation has previously been identified as a potential risk factor for growth faltering in a case control study of children 22-28 months of age from northern Tanzania, where elevated AGP levels were associated with lower HAZ [31]. In addition, a case-control study nested in a cohort study of Zimbabwean children [30] used archived plasma specimens to demonstrate higher CRP and AGP concentrations in the first year of life among children who were stunted at 18 months of age. This same study also found inverse associations between CRP and AGP and insulin-like growth factor 1 (IGF-1), a hormone that stimulates bone, muscle, and organ growth [30]. Generally, inflammation has been repeatedly associated with increased cytokine production, which in turn suppresses bone tissue formation and increases cellular destruction [32]. In other studies, inflammation has been attributed to enteropathy and gut malfunction caused by exposure to poor sanitation and hygiene conditions [33]. In contrast, our study found no associations in the multivariable models between stunting or wasting and the sanitation- or hygiene-related variables examined. Ancillary analyses also found no association between a child's inflammation status and his/her household's sanitation level (i.e., adequate vs. inadequate) or the community-level prevalence of open defecation (data not shown). The association between inflammation and community-level open defectation may be confounded by the fact that our study did not examine the population density in each surveyed community. Using data from Bangladesh, Hathi et al. [34] have found that open defecation has stronger associations with child health in high-density communities.

In this study, household food insecurity was measured using the HFIAS, and severe household food insecurity accounted for a substantial proportion of stunting in children 6-59 months of age. Psaki et al. [35] examined associations between HFIAS and child HAZ and found a significant association between higher levels of household food insecurity and lower HAZ.

Although we identified ID as a potential risk factor for stunting, the mechanism by which ID contributes to stunting is not clear. It is possible that ID may be a proxy for the lack of dietary diversity or diet poor in other micronutrients. A cross-sectional study in Indonesia found that household-level consumption of iron-rich foods, such as fish, meat, and poultry, was associated with lower prevalence of stunting among children $<5$ years of age [36].

\section{Wasting in children $0-5$ and 6-59 months of age}

As with stunting, the factors associated with wasting in children $0-5$ months and 6-59 months of age were different. In children $0-5$ months of age, the association in younger children between recent diarrhea and wasting is unsurprising. A recent systematic review of studies examining the risk factors of wasting in sub-Saharan Africa found that diarrhea was one of the most consistent factors associated wasting [37]. Diarrhea can directly 
affect nutritional status via the loss of nutrients and fluids and by reducing food intake and nutrient absorption [38]. In addition, wasting can indirectly lead to diarrhea by predisposing a child to infections by compromising his/ her immune function and increasing intestinal permeability [38]. Furthermore, concurrent wasting and diarrhea have also been shown to increase the incidence of mortality in children $<5$ years of age $[39,40]$. Our finding that no exclusively breast-fed child had recent diarrhea is consistent with, albeit somewhat more extreme than, the results of a recent meta-analysis [41]. Our multivariable modelling demonstrated independent effects of both recent diarrhea and exclusive breastfeeding on wasting; however, exclusive breastfeeding has been associated with lower prevalence of recent diarrhea and other diseases [42]. As a result, exclusive breastfeeding decreases wasting by more than one causative pathway.

Our study seems to identify an inverse association between ID and wasting. This finding is perplexing, as a recent systematic review and meta-analysis that examined the influence of low-dose iron supplementation on child growth found no association between iron status and WHZ, wasting, HAZ, or stunting [43]. Moreover, one could assume, as with stunting, that ID would denote a non-diverse diet, that could potentially make a child more likely to be wasted. ID has been shown to be protective against malaria [44], which could contribute to growth faltering [45]. However, this explanation does not relate to our findings as the 2019 SMS identified malaria in very few children $(n=9)$, none of which were wasted. Another hypothetical explanation could be that non-wasted children are fed more complementary foods containing iron-absorption inhibitors (e.g. whole grain porridge) [46]; thus improving growth whilst decreasing iron status. However, exploratory analysis of 24-h food frequency data - collected as part of our study from children 6-23 months of age - did not reveal any significant associations between wasting and consumption of grains. Alternatively, the protective effect of ID we found may suggest that there is a confounding variable not included in our analysis that is producing this spurious association.

The substantially elevated risk of wasting among the poorest children is consistent with the findings of other studies: An analysis of data from Ghana's DHS demonstrated an association between wasting and household wealth, with children in households within the poorest wealth quintile being more likely to be wasted [47]. Furthermore, a systematic review of studies on children under 5 years in sub-Saharan Africa [48] found that wasting was associated with low household wealth in Ghana, Nigeria, Ethiopia, and Kenya. Several studies across subSaharan Africa [49-51] report that higher household income and food security and greater healthcare utilization lead to improved child nutritional outcomes.

\section{Limitations}

Our analysis did not contain certain potential key risk factors for stunting and wasting. As shown in other studies, there are many variables that influence child growth and development [52]. For example, child birthweight or estimated size at birth could have been collected as indicators of growth restriction in utero. However, few children included in the 2019 SMS were weighed at birth. Further, maternal age, education, and profession have been associated with child stunting throughout subSaharan Africa region [37], but maternal risk factors of stunting and wasting could not be included in our analyses because non-pregnant women were only selected from a subsample of enrolled households. As a result, maternal information was not available for one-half of enrolled children.

Our study also did not collect stool samples from children in order to assess the presence of enteropathogens. While stool samples are rarely collected as part of national surveys, enteropathogens have been linked with child growth in other studies [53,54], and accounting for these variables would have enhanced our analyses. Dietary intake was also not included, which prevented the analysis of the associations between growth and protein intake, an association that has been observed in other studies [55]. Another limitation of our analyses is that no blood samples were taken from children 0-5 months of age, and thus, no associations between stunting and wasting and biomarker indicators, such as anemia, iron deficiency, and inflammation, could be assessed in this group. In addition, the sample size of children $0-5$ months of age was quite small, which likely affected our ability to identify significant risk factors for stunting and wasting in this age group.

Although anemia was not a significant risk factor of stunting or wasting, the use of capillary blood samples to measure hemoglobin concentration can be seen as a limitation to our study. When comparing the hemoglobin concentrations in capillary and venous blood samples in the same individuals from seven countries, Rappaport et al. [5] found consistently lower hemoglobin concentrations in the capillary samples. The authors posited that this may be due to increased levels of interstitial fluid - and conversely less hemoglobin - in capillary samples incurred through improper blood collection techniques, such as excessive squeezing of a child's finger during blood collection. While the phlebotomists were instructed to not to squeeze or "milk" a child's finger, the collection of venous blood samples would have plausibly 
increased the accuracy and precision of the hemolgobin measurements.

The standard deviations of HAZ and WHZ values are higher than those recommended by the SMART guidelines [56], which may indicate suboptimal quality of anthropometric measurements. On the other hand, these standard deviations are similar to those from DHS surveys in other African countries [57]. We observed substantial age heaping [58] which may have increased the variability of HAZ values. Despite the use of local events calendars, widespread calendar illiteracy and lack of child health cards resulted in relatively poor accuracy in the estimates of child dates of birth.

Regarding studies comparing haemoglobin results in the same individuals, a recent study by Rappaport et al. [59] combined data from 11 separate studies from seven countries with samples from children, men, nonpregnant women and pregnant women. In addition to using previously unpublished data, Rappaport et al. [59] included results from previously-published studies that compared haemoglobin measurement methods [60-65].

\section{Conclusions}

The risk factors associated with stunting and wasting differ in children $0-5$ months and 6-59 months of age, suggesting that differing interventions are required to improve child growth at various growth stages. A notable proportion of malnutrition was attributable to infection-related factors (i.e., inflammation and diarrhea), suggesting that interventions that limit a child's exposure to infection-causing pathogens would improve child growth. The dose-response associations between child growth and household food insecurity and household wealth suggest that household-level social protection interventions would have that ancillary benefit of reducing the prevalence of stunting and wasting.

\section{Abbreviations}

HFIAS: Household Food Insecurity and Access Scale; SMS: Somalia Micronutrient Survey; WHO: World Health Organization; ID: Iron deficiency.

\section{Supplementary Information}

The online version contains supplementary material available at https://doi. org/10.1186/s12889-021-12439-4.

Additional file 1: Supplementary Table S1. Prevalence of stunting by potential risk factors in children 0 - 5 months and children 6-59 months of age, Somalia 2019. Supplementary Table S2. Prevalence of wasting by potential risk factors in children 0 - 5 months and children 6-59 months of age, Somalia 2019.

\section{Acknowledgements}

We thank the parents and children who participated in the survey and the fieldworkers (national and international consultants, National/State coordinators, M \& E team leaders, field coordinators, interviewers, phlebotomists, anthropometrists, and drivers) who conducted the fieldwork. We also thank Dr. Andrew Seal (University College London) for his inputs on the study protocol and questionnaires. Lastly, we acknowledge the commitment and ownership/leadership of the Federal Ministry of Health and other federal and state-level line ministries in Somalia.

\section{Authors' contributions}

Conceptualization, F.S., F.R., N.P., J.P.W., B.A.W.; Formal analysis, J.P.W., B.A.W; Funding acquisition, F.S.; M.G.; Methodology, F.R., J.P.W., N.P., B.A.W, F. S, R.W. Project administration, F.S., J.P.W., J.M., Y.K., F.R., N.P., B.A.W., W.E.S.D., M.G., A.M.; Supervision, S.I.A., Y.K., J.M., S.M.H, K.M.M., A.M., W.S.M., A.M.M., F.M.M.; Writing-original draft, W.E.S.D., J.P.W. The author(s) read and approved the final manuscript.

\section{Funding}

The author(s) disclosed receipt of the following financial support for the research, authorship, and/or publication of this article: This analysis was conducted as part of Contract No. 43235822 between UNICEF-Somalia and GroundWork.

\section{Availability of data and materials}

The datasets used and/or analysed during the current study are available from the corresponding author on reasonable request.

\section{Declarations}

\section{Ethics approval and consent to participate}

Ethical permissions for the survey were obtained from the ethical review committees of the Federal Ministry of Health (Ref: MOH\&HS/DGO/0999/Jun/2018) and the Ministries of Health in Somaliland (Ref: MOHD/DG:2/699/2018) and Puntland (MOH/PL/DGO/0277/18).

All household heads were provided an information sheet that detailed the written informed consent process, the objectives of the survey, and the data collection procedures. This information sheet also noted that participation in the survey was voluntary. This information sheet was read aloud to all household heads in Somali or the Somaliland dialect.

Written informed consent was obtained from the household head for the administration of the survey. If the household head was illiterate, the consent form was read out loud to him/her, and a fingerprint was taken as evidence of consent instead of a signature. As part of this written informed consent form, the household head consented to a) answer the questions in the household questionnaire or permit another knowledgeable adult to answer the questions, and b) permit selected women and children in the household to participate or refuse to participate in the survey. Written informed consent for the household did not compel selected women and children within the household to participate in the survey. For children 0-59 months of age, the mother or legal guardian provided verbal consent for the child to participate in the survey. Verbal consent was also provided by the caretakers of women 15-17.9years of age; women 18-49years of age provided verbal consent for themselves. This individual-level verbal consent procedure was suggested and insisted by the ethical review committees of the Federal Ministry of Health (Ref: MOH\&HS/DGO/0999/Jun/2018) and the Ministries of Health in Somaliland (Ref: MOHD/DG:2/699/2018) and Puntland (MOH/PL/DGO/0277/18) to account for Somalia's cultural context, as they considered individual-level written informed consent culturally appropriate. Specifically, they felt the need for an individual-level written informed consent unnecessary, expressing serious concerns that household heads would not permit women to sign the consent forms, and that requiring individual-level written informed consent could potentially result in conflict within the household and limit participation in the survey. Of note, prior biomedical surveys in Somalia use verbal consent exclusively, and thus, the consent procedure used by the SMS 2019 was the most viable approach to obtain written informed consent, albeit only at the household level. This procedure to have a written informed consent, albeit only at the household level, from the household head, and verbal consent at the individual-level was approved by the ethical review committees of the Federal Ministry of Health and the Ministries of Health in Somaliland and Puntland.

If diagnosed with malaria, severe acute malnutrition, and/or severe anemia, survey participants were referred to local health facilities for treatment. No blood was taken from children younger than 6 months of age to avoid injury. 
All anthropometric and blood collection procedures, and all other methods were carried out in accordance with standard guidelines and regulations.

\section{Consent for publication}

Not applicable.

\section{Competing interests}

The authors declare no competing interests. The authors alone are responsible for the views expressed in this publication and they do not necessarily represent the decisions, policy or views of UNICEF.

\section{Author details}

${ }^{1}$ GroundWork, Hintergasse 1, 7306, Fläsch, Switzerland. ${ }^{2}$ Brandpro Ltd, Nairobi, Kenya. ${ }^{3}$ UNICEF, Mogadishu, Somalia. ${ }^{4}$ Department of Nutrition, Federal Ministry of Health, Mogadishu, Somalia. ${ }^{5}$ Department of Nutrition, Ministry of Health, Hargeisa, Somaliland. ${ }^{6}$ Department of Nutrition, Ministry of Health, Garowe, Puntland.

Received: 11 March 2021 Accepted: 15 December 2021

Published online: 09 February 2022

\section{References}

1. United Nations Children's Fund (UNICEF), World Health Organization, International Bank for Reconstruction and Development/The World Bank. Levels and trends in child malnutrition: key findings of the 2021 edition of the joint child malnutrition estimates. Geneva; 2021.

2. Victora CG, de Onis M, Hallal PC, Blossner M, Shrimpton RC. Worldwide timing of growth Faltering : revisiting implications for interventions. Pediatrics. 2020;125(3):e473-80.

3. Perkins JM, Kim R, Krishna A, McGovern M, Aguayo VM, Subramanian SV. Understanding the association between stunting and child development in low- and middle-income countries: Next steps for research and intervention. Soc Sci Med. 2017;193:101-9. https://doi.org/10.1016/j. socscimed.2017.09.039. Epub 2017 Sep 22. PMID: 29028557

4. Soekatri MYE, Sandjaja S, Syauqy A. Stunting was associated with reported morbidity, parental education and socioeconomic status in 0.5-12-year-old Indonesian children. Int J Environ Res Public Health. 2020;17(17):6204.

5. Dewey KG, Begum K. Long-term consequences of stunting in early life. Matern Child Nutr. 2011;7(s3):5-18.

6. Kinyoki DK, Berkley JA, Moloney GM, Kandala NB, Noor AM. Predictors of the risk of malnutrition among children under the age of 5 years in Somalia. Public Health Nutr. 2015;18(17):3125-33.

7. WHO. Guideline: Updates on the management of severe acute malnutrition in infants and children. Geneva world heal Organ. 2013;

8. Burki TK. Malaria and malnutrition: Niger's twin crises. Lancet. 2013;382(9892):587-8. Available from:. https://doi.org/10.1016/S0140 6736(13)61732-8.

9. United Nations World Food Programme. Food Market and Supply Situation in Southern Somalia. Rome UN-WFP. 2011; Available from: https:// documents.wfp.org/stellent/groups/public/documents/ena/wfp255737. pdf?_ga =2.155613499.1018630955.1551850909-24802211.1493311245

10. Food Security and Nutrition. Analysis Unit - Somalia. FSNAU. Post Deyr 2015/16. Technical Series Report No. VII 65. April 29, 2016. Somalia Nutrition Analysis

11. De Onis M, Borghi E, Arimond M, Webb P, Croft T, Saha K, et al. Prevalence thresholds for wasting, overweight and stunting in children under 5 years. Public Health Nutr. 2019;22(1):175-9.

12. Federal Ministry of Health, Ministry of Health [Somaliland], Ministry of Health [Puntland], UNICEF, BrandPro, GroundWork. Somalia Micronutrient Survey 2019. Mogadishu, Somalia; 2020

13. Rutstein $\mathrm{SO}$, Johnson $\mathrm{K}$. The DHS wealth index. DHS comparative reports no. 6. Calverton, Maryland: ORC Macro; 2004.

14. Filmer D, Pritchett LH. Estimating wealth effects without expenditure data - or tears. Demography; 2001.

15. WHO, UNICEF. WHO/UNICEF joint monitoring Programme for water supply, Sanitation and Hygiene (JMP) 2017.
16. Coates J, Swindale A, Bilinsky P. Household Food Insecurity Access Scale (HFIAS) for Measurement of Household Food Access: Indicator Guide (v. 3). Washington, D.C; 2007.

17. World Health Organization. Indicators for assessing infant and young child feeding practices part 2. Geneva world heal Organ. 2010;

18. Cashin K, Oot L. Guide to Anthropometry: A Practical Tool for Program Planners, Managers, and Implementers. Food Nutr Tech Assist III Proj (FANTA)/ FHI 360; 2018.

19. Cogill B. Anthropometric Indicators Measurement Guide; 2003.

20. De Onis M. WHO child growth standards based on length/height, weight and age. Acta Paediatr Int J Paediatr. 2006;95(SUPPL. 450):76-85.

21. Thurnham DI, Mburu ASW, Mwaniki DL, Muniu EM, Alumasa F, de Wagt A. Using plasma acute-phase protein concentrations to interpret nutritional biomarkers in apparently healthy HIV-1-seropositive Kenyan adults. Br J Nutr. 2008;100(1):174-82.

22. Namaste SM, Rohner F, Huang J, Bhushan NL, Flores-Ayala R, Kupka $\mathrm{R}$, et al. Adjusting ferritin concentrations for inflammation: biomarkers reflecting inflammation and nutritional determinants of Anemia (BRINDA) project. Am J Clin Nutr. 2017;106(Suppl 1):359S-71S.

23. Larson LM, Namaste SM, Williams AM, Engle-Stone R, Addo OY, Suchdev PS, et al. Adjusting retinol-binding protein concentrations for inflammation: biomarkers reflecting inflammation and nutritional determinants of Anemia (BRINDA) project. Am J Clin Nutr. 2017;106(Suppl 1):390S-401S.

24. Stewart CP, lannotti L, Dewey KG, Michaelsen KF, Onyango AW. Contextualising complementary feeding in a broader framework for stunting prevention. Matern Child Nutr. 2013;9(S2):27-45.

25. STATA. Nptrend - test for trend across ordered groups. STATA manual 2020.

26. Rockhill B, Newman B, Weinberg C. Use and misuse of population attributable fractions. Am J Public Health. 1998;88(1):15-9.

27. WHO. In: Organization WH, editor. Nutrition landscape information system (NLIS): country profile indicators - interpretation guide. Geneva: World Health Organization; 2010.

28. Danaei G, Andrews KG, Sudfeld CR, Fink G, McCoy DC, Peet E, et al. Risk factors for childhood stunting in 137 developing countries: a comparative risk assessment analysis at global, regional, and country levels. PLoS Med. 2016;13(11):e1002164.

29. Syed S, Manji KP, McDonald CM, Kisenge R, Aboud S, Sudfeld C, et al. Biomarkers of systemic inflammation and growth in early infancy are associated with stunting in young Tanzanian children. Nutrients. 2018 Aug;10(9):1158.

30. Prendergast AJ, Rukobo S, Chasekwa B, Mutasa K, Ntozini R, Mbuya MNN, et al. Stunting is characterized by chronic inflammation in zimbabwean infants. PLoS One. 2014;9(2):e86928.

31. Wirth JP, Kitilya B, Petry N, PrayGod G, Veryser S, Mngara J, et al. Growth status, inflammation, and enteropathy in young children in northern Tanzania. Am J Trop Med Hyg. 2019;100(1):192-201.

32. Millward DJ. Nutrition, infection and stunting: the roles of deficiencies of individual nutrients and foods, and of inflammation, as determinants of reduced linear growth of children. Nutr Res Rev. 2017;30(1):50-72.

33. Humphrey JH. Child undernutrition, tropical enteropathy, toilets, and handwashing. Lancet. 2009;374(9694):1032-5.

34. Hathi P, Haque S, Pant L, Coffey D, Spears D. Place and child health: the interaction of population density and sanitation in developing countries. Demography. 2017:54(1):337-60.

35. Psaki S, Bhutta ZA, Ahmed T, Ahmed S, Bessong P, Islam M, et al. Household food access and child malnutrition: results from the eight-country MAL-ED study. Popul Health Metrics. 2012;10:24.

36. Mahmudiono T, Sumarmi S, Rosenkranz RR. Household dietary diversity and child stunting in East Java, Indonesia. Asia Pac J Clin Nutr. 2017;26(2):317-25.

37. Akombi BJ, Agho KE, Hall JJ, Wali N, Renzaho AMN, Merom D. Stunting, wasting and underweight in sub-Saharan Africa : a systematic review. Int J Env Res Public Heal. 2017;14(8):1-18.

38. Brown KH. Diarrhea and malnutrition. J Nutr. 2003.

39. Masanja H, Baraka J, Jackson B, Manji K, Iriya N, Kimatta S. Factors associated with the decline in under five diarrhea mortality in Tanzania from 1980-2015. J Glob Health. 2019;9(2):1-9. 
40. Troeger C, Forouzanfar M, Rao PC, Khalil I, Brown A, Reiner RC, et al. Estimates of global, regional, and national morbidity, mortality, and aetiologies of diarrhoeal diseases: a systematic analysis for the global burden of disease study 2015. Lancet Infect Dis. 2017;17(9):909-48.

41. Wijiwinarsih A, Susilawati TN, Murti B. The effect of exclusive breastfeeding on wasting in children under five: a Meta-analysis study. J Matern Child Heal. 2019;4:87-96.

42. Saeed OB, Haile ZT, Chertok IA. Association between exclusive breastfeeding and infant health outcomes in Pakistan. J Pediatr Nurs. 2020;50:e62-8.

43. Petry N, Olofin I, Boy E, Angel MD, Rohner F. The effect of low dose Iron and zinc intake on child micronutrient status and development during the first 1000 days of Life : a systematic review. Nutrients. 2016;8(12):773.

44. Clark MA, Goheen MM, Fulford A, Prentice AM, Elnagheeb MA, Patel J, et al. Host iron status and iron supplementation mediate susceptibility to erythrocytic stage plasmodium falciparum. Nat Commun. 2014;5:4446.

45. Lee G, Yori P, Olortegui MP, Pan W, Caulfield L, Gilman RH, et al. Comparative effects of vivax malaria, fever and diarrhoea on child growth. Int J Epidemiol. 2012;41(2):531-9.

46. Gibson RS, Bailey KB, Gibbs M, Ferguson EL. A review of phytate, iron, zinc, and calcium concentrations in plant-based complementary foods used in low-income countries and implications for bioavailability. Food Nutr Bull. 2010;31(2_suppl2):S134-46.

47. Darteh EKM, Acquah E, Darteh F. Why are our children wasting: Determinants of wasting among under 5s in Ghana. Nutr Health. 2017;23(3):15966. https://doi.org/10.1177/0260106017722924. Epub 2017 Aug 4. PMID: 28774231

48. Akombi BJ. Stunting, Wasting and Underweight in Sub-Saharan Africa : A Systematic Review. Int J Env Res Public Heal. 2017;14(8):1-18.

49. Adekanmbi VT, Kayode GA, Uthman OA. Original Article Individual and contextual factors associated with childhood stunting in Nigeria : a multilevel analysis. Matern Child Nutr. 2013;9(2):244-59.

50. Aheto JMK, Keegan TJ, Taylor BMDP. Childhood malnutrition and It's determinants among under-five children in Ghana. Paediatr Perinat Epidemiol. 2015;29(6):552-61.

51. Chirande L, Charwe D, Mbwana H, Victor R, Kimboka S, Issaka Al, et al. BMC Pediatr [Internet]. 2015:1-13. Available from:. https://doi.org/10. 1186/s12887-015-0482-9.

52. Woodruff BA, Wirth JP, Bailes A, Matji J, Timmer A, Rohner F, et al. Matern Child Nutr. 2017;13(2):1-17.

53. Donowitz JR, Pu Z, Lin Y, Alam M, Ferdous T, Shama T, Taniuchi M, Islam MO, Kabir M, Nayak U, Faruque ASG, Haque R, Ma JZ, Petri WA Jr. Small Intestine Bacterial Overgrowth in Bangladeshi Infants Is Associated With Growth Stunting in a Longitudinal Cohort. Am J Gastroenterol. 2021;117(1):167-75. https://doi.org/10.14309/ajg.0000000000001535. Epub ahead of print. PMID: 34693912; PMCID: PMC8715995.

54. Owino V, Ahmed T, Freemark M, Kelly P, Loy A, Manary M, et al. Environmental enteric dysfunction and growth failure/stunting in global child health. Pediatrics. 2016;138(6):e20160641.

55. Fikawati S, Syafiq A, Ririyanti RK, Gemily SC. Energy and protein intakes are associated with stunting among preschool children in Central Jakarta, Indonesia: a case-control study. Malays. J Nutr. 2021;27(1):081-91. https:// doi.org/10.31246/min-2020-0074.

56. Smart Manual. SMART (standardized monitoring and assessment for relief and transitions) Manual 2.0. Action againt Hunger Tech Advis Gr; 2017. p. $1-146$.

57. Mei G-SLM. Standard deviation of anthropometric Z-scores as a data quality assessment tool using the 2006 WHO growth standards: a cross country analysis. Bull World Health Organ. 2007;85(6):441-8.

58. GroundWork, Brandpro, Ministry of Health, UNICEF. 2019 Somalia National Nutrition Survey. Mogadishu, Somalia; 2020.

59. Rappaport Al, Karakochuk CD, Hess SY, Whitehead RD Jr, Namaste SML, Dary O, Parker ME, Neufeld LM, Larson LM, Newton S, Wegmuller R, Moorthy D. Variability in haemoglobin concentration by measurement tool and blood source: an analysis from seven countries. J Clin Pathol. 2021;74(10):657-63. https://doi.org/10.1136/jclinpath-2020-206717. Epub 2020 Oct 6. PMID: 33023940

60. Karakochuk CD, Janmohamed A, Whitfield KC, Barr SI, Vercauteren SM, Kroeun $\mathrm{H}$, et al. Evaluation of two methods to measure hemoglobin concentration among women with genetic hemoglobin disorders in Cambodia: a method-comparison study. Clin Chim Acta. 2015;441:148-55.
61. Rappaport Al, Karakochuk CD, Whitfield KC, Kheang KM, Green TJ. A method comparison study between two hemoglobinometer models (Hemocue Hb 301 and $\mathrm{Hb}$ 201+) to measure hemoglobin concentrations and estimate anemia prevalence among women in Preah Vihear, Cambodia. Int J Lab Hematol. 2017;39(1):95-100.

62. Whitehead RD, Zhang M, Sternberg MR, Schleicher RL, Drammeh B, Mapango $C$, et al. Effects of preanalytical factors on hemoglobin measurement: a comparison of two HemoCue ${ }^{\circledR}$ point-of-care analyzers. Clin Biochem. 2017:50(9):513-20.

63. Neufeld LM, Larson LM, Kurpad A, Mburu S, Martorell R, Brown KH. Hemoglobin concentration and anemia diagnosis in venous and capillary blood: biological basis and policy implications. Ann NY Acad Sci. 2019;1450(1):172-89.

64. Hinnouho G-M, Barffour MA, Wessells KR, Brown KH, Kounnavong S, Chanhthavong $B$, et al. Comparison of haemoglobin assessments by HemoCue and two automated haematology analysers in young Laotian children. J Clin Pathol. 2017; jclinpath-2017-204786.

65. Karakochuk CD, Barker MK, Whitfield KC, Barr SI, Vercauteren SM, Devlin $A M$, et al. The effect of oral iron with or without multiple micronutrients on hemoglobin concentration and hemoglobin response among nonpregnant Cambodian women of reproductive age: a $2 \times 2$ factorial, double-blind, randomized controlled supplementation trial. Am J Clin Nutr. 2017;106(1):233-44.

\section{Publisher's Note}

Springer Nature remains neutral with regard to jurisdictional claims in published maps and institutional affiliations.
Ready to submit your research? Choose BMC and benefit from:

- fast, convenient online submission

- thorough peer review by experienced researchers in your field

- rapid publication on acceptance

- support for research data, including large and complex data types

- gold Open Access which fosters wider collaboration and increased citations

- maximum visibility for your research: over $100 \mathrm{M}$ website views per year

At BMC, research is always in progress.

Learn more biomedcentral.com/submissions 